\title{
Investigation of the Application of a Two-Wavelength Mach-Zehnder Interferometer to Measure Soret Coefficients
}

\author{
By Yuhi Mori ${ }^{1}$, Yoshitaka Hashimoto $^{1)}$, Shinsuke SUZUKI ${ }^{1)}$ and Yuko INATOMI $^{2)}$ \\ 1) Department of Applied Mechanics, Waseda University, Tokyo, Japan \\ ${ }^{2)}$ Institute of Space and Astronautical Science, Japan Aerospace Exploration Agency, Sagamihara, Japan
}

(Received June 24th, 2013)

\begin{abstract}
We developed a measurement method of Soret coefficient $S_{T}$ using a two-wavelength Mach-Zehnder interferometer. Temperature gradient was applied to pure molten salol or the salol - $6 \mathrm{~mol} \%$ tert-butyl alcohol solution in a quartz cell. The direction of the temperature gradient was opposite to the gravity vector so as to suppress the convection. The movement of the interference fringes in solution side was evaluated by the number of the dark lines crossing a measuring point from right to left. The changes of temperature and concentration were measured using the number of the dark lines. Wavy noise found in measured number of the dark lines could be removed by subtracting one in the cell wall. The temperature change agreed with one measured using thermocouples. The results show that the measurement method in this study enables the contactless $S_{T}$ measurement.
\end{abstract}

Key Words: Soret Effect, Thermal Diffusion, Mach-Zehnder Interferometer, ISS, JEM

$\begin{array}{cll}\text { Nomenclature } & \\ \lambda & : & \text { wavelength of light source } \\ \Delta m & : & \text { the number of the dark lines crossing a } \\ & & \text { measurement point } \\ n & : & \text { refractive index } \\ c & : & \text { thickness of sample liquid } \\ T & : & \text { temperature } \\ C & : & \text { mole fraction of tert-butyl alcohol } \\ S_{T} & : & \text { Soret coefficient } \\ D & : & \text { diffusion coefficient } \\ D_{T} & : & \text { thermal diffusion coefficient } \\ X_{C} & : & \text { distance from the centerline of cell } \\ X_{T} & : & \text { distance from the upper surface of cell } \\ t & : & \text { elapsed time since starting to give } \\ & & \text { difference of temperature } \\ \text { Subscripts } & & \\ 0 & : & \text { initial state } \\ \text { W } & : & \text { wall of quartz cell } \\ \mathrm{S} & : & \text { solution } \\ 532 & : & 532 \text { nm, wavelength of laser beam } \\ 780 & : & 780 \text { nm, wavelength of laser beam }\end{array}$

\section{Introduction}

When a temperature gradient is applied to mixed solution having uniform solute concentration, the solute is transported to either cold or hot side and a solute concentration gradient is established. This phenomenon is called Soret effect (thermal diffusion). This thermodynamic indicator, Soret coefficient $S_{T}$, is expressed by the ratio of diffusion coefficient $D$ and thermal diffusion coefficient $D_{T}$ as Eq. (1).

$$
S_{T}=\frac{D_{T}}{D}
$$

Soret effect plays an important role to redistribute solutes during crystal growth of alloy ${ }^{1)}$. Thus, development of a measurement method for Soret coefficients $S_{T}$ with high accuracy is required.

Soret cell is known as the classical measurement method for $S_{T}$ of mixed solutions ${ }^{2)}$. The Soret cell consists of the two thin plates sandwiching a sample solution film. The gap between the plates is maintained by braces. The braces have holes to extract the solution using a syringe to measure the solute concentration gradient. However, this sampling method using syringe is an error source for concentration measurements because of forced convective disturbance during extracting the solution. Therefore, interferometry has been applied to the measurement of $S_{T}$ so as to avoid forced convective disturbance ${ }^{3-7)}$, because interferometry enables contactless measurement of either concentration or temperature. Moreover, space environments have been utilized in order to avoid natural convective disturbance. Shevtsova et al. ${ }^{8)}$ measured diffusion coefficient and Soret coefficient of binary mixed solution using one-wavelength Mach-Zehnder interferometer in the ESA Columbus module of the International Space Station (ISS) (the Influence of Vibrations on Diffusion in Liquids (IVIDIL) mission). However, a one-wavelength Mach-Zehnder interferometer does not enable the contactless simultaneous measurement of both concentration and temperature of binary solution. The temperature of sample solution was estimated from the measured temperature of the copper block. This way of temperature estimation could be one of the error sources.

We are planning to measure the Soret coefficient of binary mixed solution using a two-wavelength Mach-Zehnder 
interferometer in the Japanese Experiment Module (JEM) "Kibo" of the ISS (the Soret-Facet mission). Two-wavelength Mach-Zehnder interferometer enables the contactless simultaneous measurement of both concentration and temperature of binary solution ${ }^{9)}$. The salol - tert-butyl alcohol alloy, which was used in the Facet mission ${ }^{10)}$, is used as sample solution in the Soret-Facet mission, too.

The present study was done to evaluate the feasibility of the Soret-Facet mission. The possibility of the temperature and concentration measurement for Soret coefficient using two-wavelength Mach-Zehnder interferometer was confirmed by the experiments.

\section{Experimental}

Pure salol (phenyl salicylate, Wako Yakuhin Inc.) and a salol - 6 mol\% tert-butyl alcohol (98 mass\%, Tokyo Chemical Industry Co.) solution were used as sample liquids. Especially, salol was purified by several zone-melting cycles in order to eliminate errors from impurities.

Two kinds of transparent quartz cells were used as the containers for the samples (Fig. 1). The cell for pure salol was equipped with two holes for inserting K-type thermocouples (TC). The temperatures of pure salol were measured using the thermocouples and recorded every 10 seconds with data logger (CT05SD, CUSTOM Ins.) so as to compare with the measurement results of the interferometer. The gaps around the TC in the holes were sealed with silicon rubber (KE45, Shin-Etsu Chemical Co.) to prevent the pure molten salol from leaking out. On the other hand, the cell for the salol solution has no holes so that volatile tert-butyl alcohol can be sealed tightly.

Figure 2 shows the two-wavelength Mach-Zehnder interferometer with wavelength $\lambda$ of $532 \mathrm{~nm}$ and $780 \mathrm{~nm}$ diode lasers (84928 and 83840, respectively, Edmund Optics Inc.) for the measurement of variation in the temperature $\Delta T$ and concentration $\Delta C$. A laser beam of either $532 \mathrm{~nm}$ or $780 \mathrm{~nm}$, which was irradiated alternatively with a switching plate every 3 seconds, passed through spatial filter, beam splitter and lens. When the laser beam reached the first half miller, the beam split into two lines with and without the quartz cell. Finally, the both beams overlapped each other and interference fringe was generated because of the optical path difference between two lines.

The temperatures of Peltier devices were controlled by feeding back the temperatures of aluminum blocks using Peltier device controller (Fig. 2) following the temperature program as shown in Fig. 3. At first, both top and bottom sides of the cell were heated at $60^{\circ} \mathrm{C}$ with Peltier devices to melt the sample liquid and homogenize the temperature and concentration of the sample liquid. The temperature was set lower than $60^{\circ} \mathrm{C}$ so as to avoid evaporation of sample. Over 12 hours later from turning on the Peltier devices, we started taking movies of variation in a certain dark line of interference fringes with a CCD video camera (MS-58H, MOSWELL Co.) and saved them to a hard disk drive. The positions of viewing fields were set as shown in Fig. 1. Soon after that, the bottom side was cooled from $60^{\circ} \mathrm{C}$ to $30^{\circ} \mathrm{C}$. Now, $t$ stands for the elapsed time since starting to give difference of temperature. A positive density gradient by the negative temperature gradient to the gravity vector was applied to avoid natural convective disturbance.

The movement of the interference fringes in solution side was evaluated by the number of the dark lines crossing a measuring point from the right to the left (Fig. 4). The symbol $L$ stands for the distance of the travelled dark line (black arrow) per unit time from measuring point and $W$ stands for the width of the fringe. The number of dark lines crossing the measuring point $\Delta m$ was calculated by Eq. (2).

$$
\Delta m=L / W
$$

The change of fringe pattern depends on the change of refractive index of sample liquid. Thus, the change of refractive index $\Delta n$ is expressed by Eq. (3).

$$
\Delta n=\frac{\lambda}{c} \Delta m
$$

Here $c$ is the thickness of sample liquid (=1 mm); $\Delta m$ is the number of dark line crossing a measuring point. The refractive index of the sample liquid depends on both the temperature and concentration of sample liquid.

$$
\Delta n=\frac{\partial n}{\partial T} \Delta T+\frac{\partial n}{\partial C} \Delta C
$$

Here $\partial n / \partial T$ and $\partial n / \partial C$ represent the dependence of refractive index on temperature and solute concentration, respectively. Combining Eq. (3) and Eq. (4), Eqs. (5a) and (5b) are obtained.

$$
\begin{gathered}
\left(\begin{array}{c}
\Delta T \\
\Delta C
\end{array}\right)=A\left(\begin{array}{l}
\Delta m_{532} \\
\Delta m_{780}
\end{array}\right) \\
A=\left(\begin{array}{cc}
1.965 & -4.519 \\
-1.238 & 1.847
\end{array}\right)
\end{gathered}
$$

Here matrix $\boldsymbol{A}$ is based on the refractive index depending on temperature and tert-butyl alcohol concentration in the salol solution. The values of matrix $\boldsymbol{A}$ are the measurement data obtained for Facet mission by JAXA (not yet published).

Now, assuming that sample solution has linier temperature and concentration gradient, Soret coefficient $S_{T}$ is calculated by following Eq. (6) using the measured $\Delta T$ and $\Delta C$.

$$
S_{T}=-\frac{\Delta C / X_{C}}{C_{0}\left(1-C_{0}\right) \Delta T / X_{T}}
$$

Here $X_{C}$ is defined as the distance from the centerline between the upper surfaces and bottom interface of the quartz cell, and $X_{T}$ is defined as the distance from the upper surface (Fig. 1). Table 1 shows the values of $X_{T}$ and $X_{C}$. 


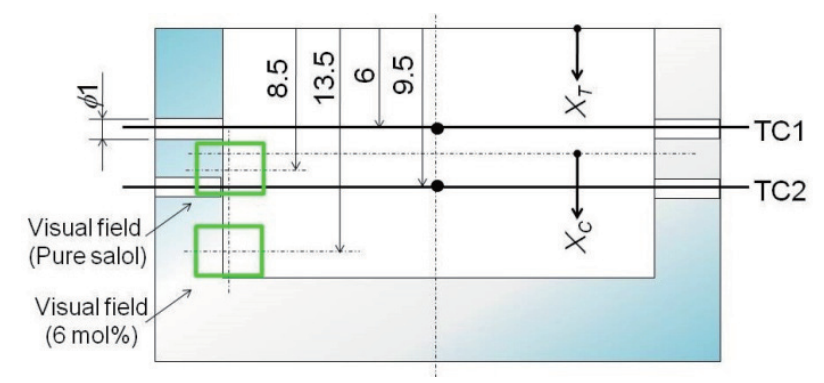

Fig. 1. Schematic illustration of the quartz cell. The cell for the experiments using pure salol has holes and thermocouple 1, 2 (TC1, TC2). On the other hands, the cell for the experiments using salol - $6 \mathrm{~mol} \%$ tert-butyl alcohol solution has no holes.

Table 1. Values of $X_{T}$ and $X_{C}$

\begin{tabular}{|l|c|c|}
\hline & $X_{T}$ & $X_{C}$ \\
\hline Pure molten salol & $8.5 \mathrm{~mm}$ & \\
\hline Salol - 6 mol\% tert-butyl alcohol solution & $13.5 \mathrm{~mm}$ & $6 \mathrm{~mm}$ \\
\hline
\end{tabular}

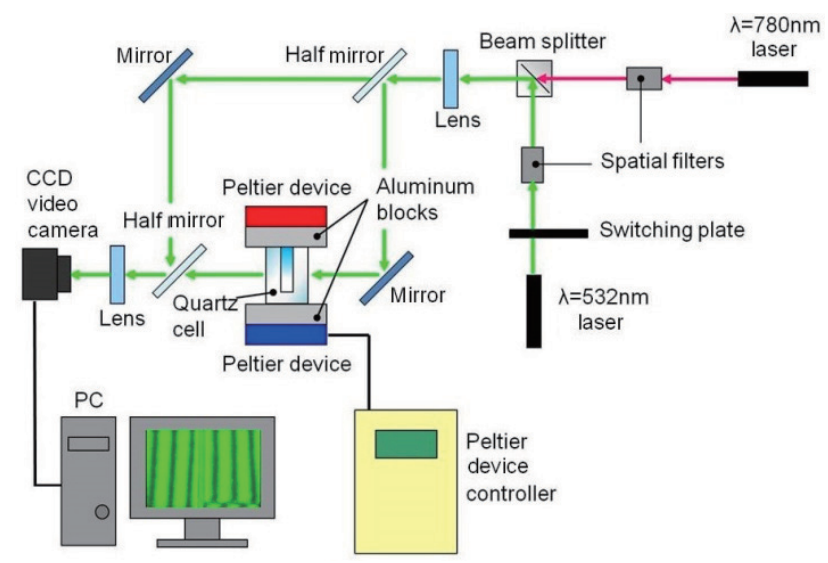

Fig. 2. Schematic illustration of the two-wavelength Mach-Zehnder interferometer.

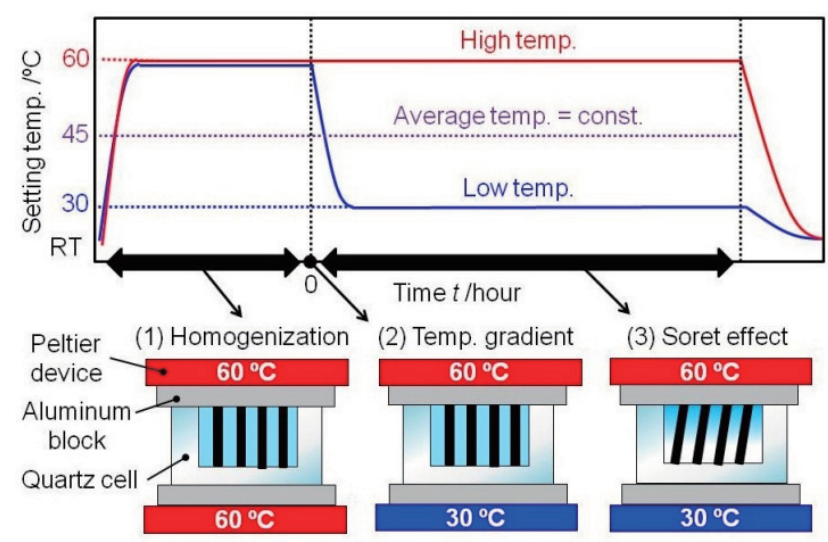

Fig. 3. Setting temperature program of Peltier devices.

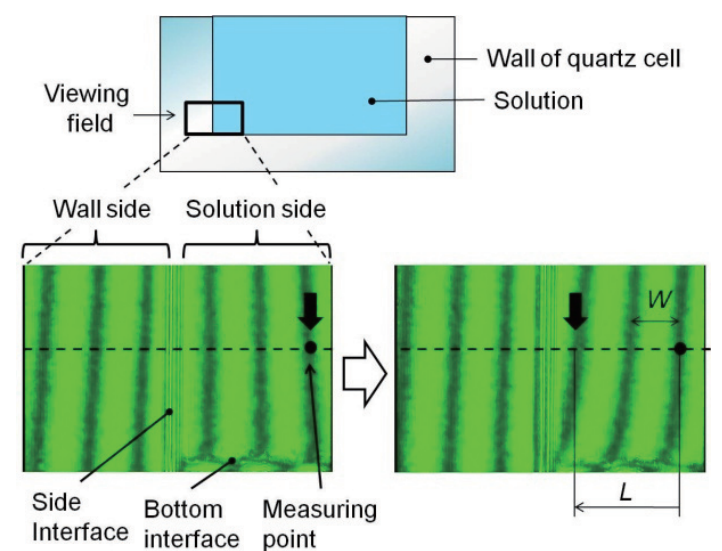

(a) Before $t=0 \mathrm{~h}$

(b) Just after $\boldsymbol{t}=\mathbf{0} \mathrm{h}$

Fig. 4. Example of shift of the interference fringe (532 nm laser). The dark lines of solution side start to move parallel to left with getting slanted at $t=0 \mathrm{~h}$.

\section{Results and Discussion}

Figure 5 shows the variations in the number of the crossing dark lines $\Delta m$ of pure molten salol using Mach-Zehnder interferometer with only $532 \mathrm{~nm}$ diode laser. The symbols $\Delta m_{s}$ and $\Delta m_{w}$ stand for the $\Delta m$ in solution side and in the wall side, respectively. When $\Delta m$ is a positive value, the dark lines move from the right to the left in Fig. 4. Both $\Delta m_{s}$ and $\Delta m_{w}$ have the similar wavy pattern with a period of about 30 hours. Now, assuming that the wavy pattern does not depend on the temperature of pure molten salol, the $\Delta m$ depending on only the melt temperature is expressed by subtracting $\Delta m_{w}$ from $\Delta m_{s}$. Fig. 6 shows the comparison between the results of calculation $\Delta T_{532}$ by Eq. (5) using $\Delta m_{532}$ and measurements using TC1 and TC2. In Fig. 6, $\Delta T_{532}$ is lying between the results of the melt temperature measurement using TC1 and TC2 (Fig. 1). Therefore, the wavy pattern does not depend on the temperature of pure molten salol and can be removed by subtracting $\Delta m_{w}$ from $\Delta m_{s}$.

Then, $\Delta T$ and $\Delta C$ were calculated by Eq. (5) using $\Delta m_{532}$ and $\Delta m_{780}$ of salol - $6 \mathrm{~mol} \%$ tert-butyl alcohol solution in order to obtain Soret coefficient (Fig. 7). The curve of the variation in concentration $\Delta C$ has a spike around $t=6 \mathrm{~h}$ in Fig. 7. There is some possibility that the spike was caused by unexpected mechanical vibration with a limited period and does not depend on Soret coefficient. If the mass transfer resulted in Soret effect became steady at $t=8 \mathrm{~h}, S_{T}$ was calculated as $8.8 \pm 0.81 \times 10^{-3} \mathrm{~K}^{-1}\left(\Delta S_{T} / S_{T}=9.2 \%\right)$ by Eq. (6) using the average of the $\Delta T$ and $\Delta C$ after $t=8 \mathrm{~h}$ in Fig. 7. Here $\Delta S_{T}$ is the standard deviation among the several $S_{T}$ values obtained after $t=8 \mathrm{~h}$. If convective disturbance is not avoided, the concentration gradient should be smaller, which makes the measured value of $S_{T}$ smaller than the real one. Therefore, the microgravity experiment is necessary to examine the reliability of the $S_{T}$ obtained under the terrestrial condition. 


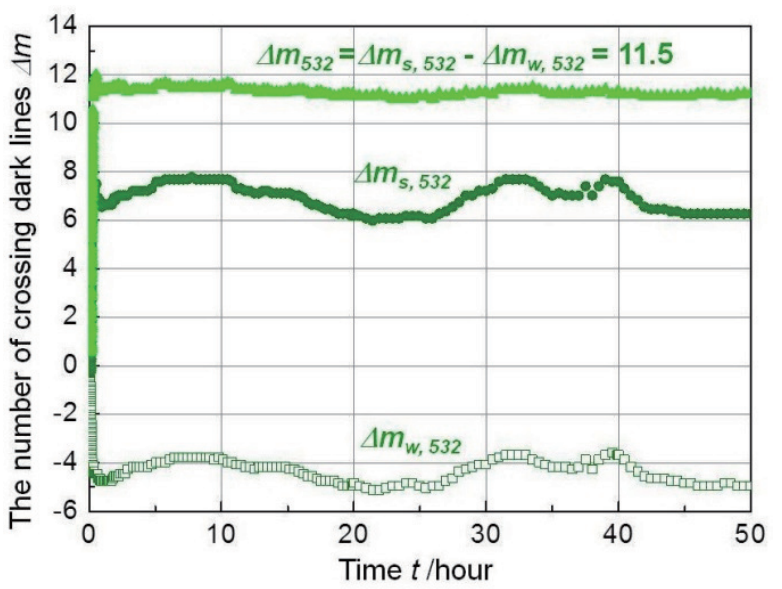

Fig. 5. The number of crossing dark lines $\Delta m$ of pure molten salol against time $t$.

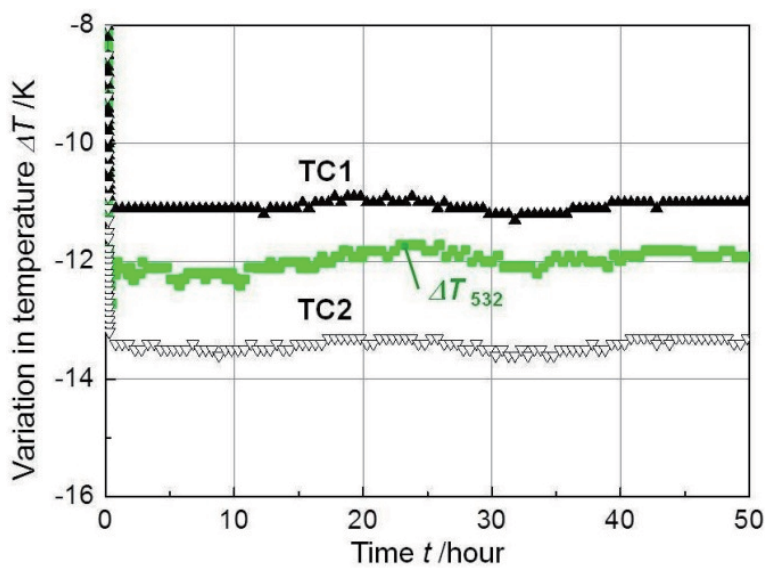

Fig. 6. Comparing the measuring results about the variations in $\Delta T$ of pure molten salol with thermocouples and with the interferometer against time $t$.

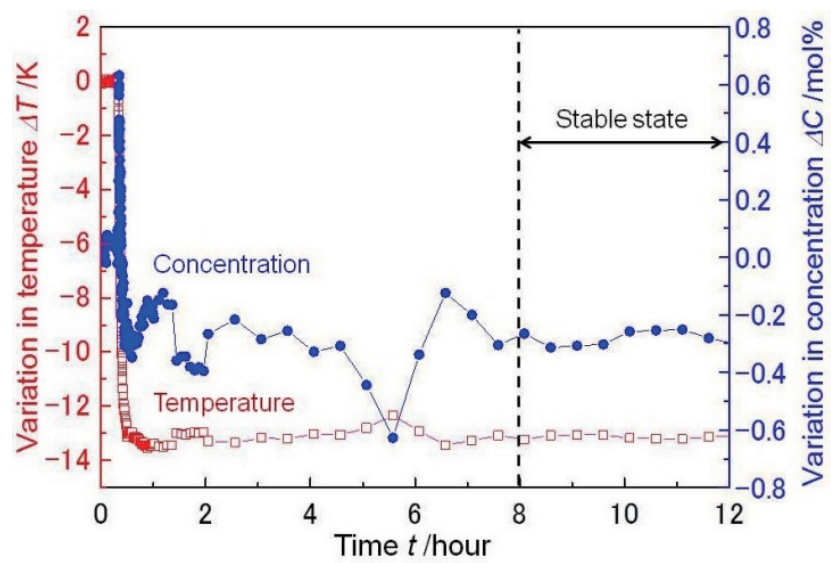

Fig. 7. Variations in $\Delta T$ and $\Delta C$ of a salol - $6 \mathrm{~mol} \%$ tert-butyl alcohol solution against time $t$.

\section{Conclusions}

We measured the number of crossing dark lines of interference fringes $\Delta m$ of pure molten salol and the salol - 6 mol\% tert-butyl alcohol solution using two-wavelength Mach-Zehnder interferometer under the condition of given temperature gradient to measure Soret coefficient $S_{T}$. We clarified that the interferometer enables the measurement of $S_{T}$ and reached the following conclusions.

1. The $\Delta m$ measured by two-wavelength Mach-Zehnder interferometer has the wavy noises with a period of about 30 hours, which can be removed by subtracting $\Delta m_{w}$ from $\Delta m_{s}$.

2. The variation in temperature $\Delta T$ and concentration $\Delta C$ can be measured by two-wavelength Mach-Zehnder interferometer. Therefore, $S_{T}$ can be calculated using the interferometer.

3. The variations in temperature $\Delta T$ and in concentration $\triangle C$ become steady at $t=8 \mathrm{~h}$.

4. The measured Soret coefficient $S_{T}$ is as follow; $S_{T} \pm \Delta S_{T}=8.8 \pm 0.81 \times 10^{-3} \mathrm{~K}^{-1}, \Delta S_{T} / S_{T}=9.2 \%$.

\section{Acknowledgments}

We are grateful to the Japan Aerospace Exploration Agency (JAXA), the Japan Space Forum (JSF) and Diffusion WG for technical advice and financial assistance throughout this investigation. We thank Kimura Chuzosho Co., EKK Japan Co. and the Special Coordination Funds for Promoting Science and Technology "Waseda Institute for Advanced Study Tenure Track Program" (Japan Science and Technology Agency) for financial assistance throughout this investigation.

\section{References}

1) Verhoeven, J. D.: The effect of thermal diffusion on constitutional supercooling during solidification, Scr. Metall., 1 (1967), pp. 93-96.

2) Costesèque, P., Pollak, T., Platten, J. K. and Marcoux, M. Transient-state method for coupled evaluation of Soret and Fick coefficients, and related tortuosity factors, using free and porous packed thermodiffusion cells: Application to $\mathrm{CuSO} 4$ aqueous solution, Eur. Phys. J., E15 (2004), pp. 249-253.

3) Mialdun, A. and Shevtsova, V.: Development of optical digital interferometry technique for measurement of thermodiffusion coefficients, Int. J. Heat and Mass Trans., 51 (2008), pp. 3164-3178.

4) Mialdun, A. and Shevtsova, V.: Measurement of the Soret and diffusion coefficients for benchmark binary mixtures by means of digital interferometry, J. Chem. Phys., 134 (2011), pp.1-12.

5) Kolodner, P., Williams, H. and Moe, C.: Optical measurement of the Soret coefficient of ethanol/water solutions, J. Chem. Phys., 88 (1988), pp. 6512-6524.

6) Zhang, K. J., Briggs, M. E., Gammon, R. W. and Sengers, J. V.: Optical measurement of the Soret coefficient and the diffusion coefficient of liquid mixtures, J. Chem. Phys., 104 (1996), pp. 6881-6892.

7) Kita, R., Wiegand, S. and Luettmer-Strathmann, J.: Sign change of the Soret coefficient of poly (ethylene oxide) in water/ethanol mixtures observed by thermal diffusion forced Rayleigh scattering, J. Chem. Phys., 121 (2004), pp. 3874-3885.

8) Shevtsova, V.: IVIDIL experiment onboard the ISS, Adv. Space Res., 46 (2010), pp. 672-679.

9) Inatomi, Y., Kuribayashi, K., Kawasaki, K. and Yoda, S.: In situ observation of unidirectional dissolution process in organic alloy under microgravity, J. Jpn. Soc. Microgravity Appl., 10 (1993), pp. 234-240.

10) Inatomi, Y., Yoshizaki, I., Sakata, K., Shimaoka, T., Sone, T., Tomobe, T., Adach, S., Yoda, S. and Yoshimura, Y.: Investigation on mechanism of faceted cellular array growth in International Space Station, Defect Diffus. Forum, 323-325 (2012), pp. 533-537. 